\title{
Iterative dummy area method for the improvement of CGH reconstruction using angler spectrum method
}

\author{
Yusei Uezono a,"* Kouki Nakashima $^{\mathrm{b}}$, Shiyuan Yang ${ }^{\mathrm{a}}$ \\ ${ }^{a}$ Kyusyu Institute of Technology, 1-1 Sensui-cho Tobata-ku Kitakyusyu-shi Fukuoka-ken, 804-8550, Japan \\ *Corresponding Author: yang@elcs.kyutech.ac.jp
}

\begin{abstract}
In this paper, we describe an iterative dummy area method for the improvement of computer-generated hologram (CGH) reconstruction using angler spectrum method. A hologram is a medium on which interference fringes of light are recorded. It is expected not only to represent three dimensions but also to be applied in various applications such as storage.

Hologram has two steps of production and reconstruction. A CGH distribution is calculated by computer. We suggest a method to reduce the reconstruction error of $\mathrm{CGH}$ using a dummy area added to the original image. It is known that if the dummy area is added to the input image, the error of the reconstructed image can be reduced. The size of the dummy area can be specified. As the area is set to be larger, the error of the reconstructed image can be reduced, there arises a problem that the size of the hologram increases. The size of the hologram should not be large because it depends on the output device. In order to solve this problem, we consider an appropriate setting method of the dummy area. Therefore, we can expect to improve the resolution of the reconstructed image without changing the size of the hologram using the iterative dummy area method.
\end{abstract}

Keywords: angler spectrum method, iterative dummy area method.

\section{Computer generated hologram (CGH)}

\subsection{Holography}

Techniques for recording / reproducing light (object light) from an object by using interference / diffraction of light. It is possible to record not only the intensity of light but also the phase of light
The medium recorded using this technology is called a hologram

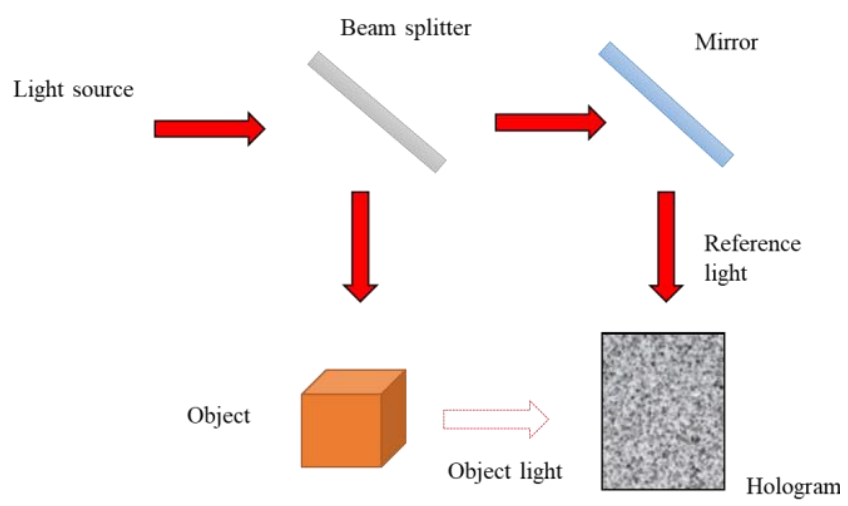

Fig. 1.1 Creation of hologram.

First, explain how to produce a hologram Light interference is used for holography recording. Split the light in two directions. One towards the object and the other towards the recording material. The light reflected from the object is called object light. The light going to the recording material is called reference light. At this time, Light interference occurs in the recording material and a hologram is created.

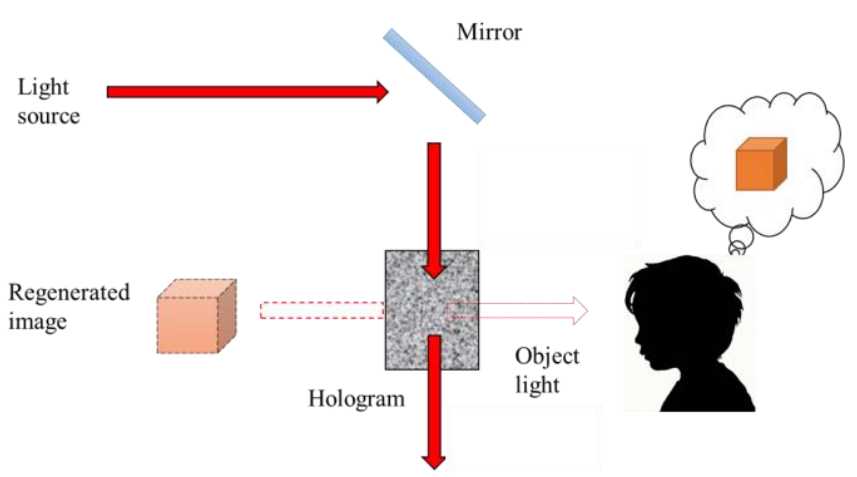

Fig. 1.2 Reconstruction of hologram.

The following illustrates the method of reconstruction hologram Light diffraction is used for reconstruction. When light is irradiated on the hologram in the same manner as 
when it is created. The observer recognizes that the recorded object exists beyond the hologram.

\subsection{Optical hologram and CGH}

In case of optical hologram when it is not CGH. To summarize the hologram creation described in the previous section. Object, light source, recording material Set up, Light interference occurs. Create a hologram. This work is very detailed and difficult

In $\mathrm{CGH}$, this process is calculated by computer. In this way, there is no need to actually set up light sources or objects and interfere with light. In addition, the calculation uses a method called an angular spectrum method

\section{Angler spectrum method}

\section{1 angular spectrum method}

There are several ways to calculate light propagation in CGH. I use angular spectrum method for calculation. This method uses FFT (Fast Fourier Transform). Therefore, calculation is fast. In addition to that, it is not subject to the distance constraint when reproducing the hologram.

The angular spectral method is performed using two Fourier transformations as shown in equation (1)

$$
\begin{aligned}
& U(u, v) \\
& =\mathcal{F}^{-1}\left[\mathcal{F}\{g(x, y)\} \exp \left(j k z \sqrt{1-\left(\lambda f_{x}\right)^{2}-\left(\lambda f_{y}\right)^{2}}\right)\right]
\end{aligned}
$$

\section{$g(x, y)$ : Complex amplitude distribution of propagation} source

$U(u, v)$ : Complex amplitude distribution at the propagation destination

: the magnitude of the wavenumber vector

: Original image - distance between holograms

$\lambda$ : Wavelength of the light source

$f_{x}$ : Spatial frequency

$\mathcal{F}, \mathcal{F}^{-1}$ : Fourier transform, inverse transform

\subsection{Production of hologram}

I will explain the procedure of creating a hologram using the angular spectrum method

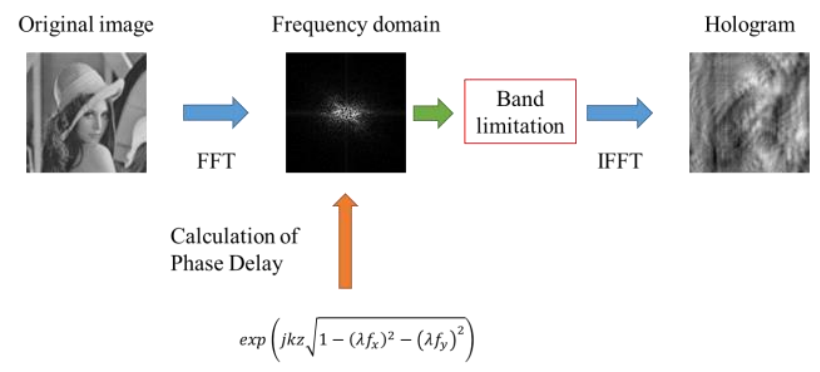

Fig. 2.1 Production of hologram.

First, Fourier transform the original image and calculate the phase delay amount in the frequency domain.

Here, the sampling theorem is satisfied, band limitation is performed to prevent aliasing

We create a hologram by performing inverse Fourier transform on this.

\subsection{Simulation of hologram reconstruction}

Next, we will explain the procedure for simulating hologram reconstruction using the angler spectrum method on a computer

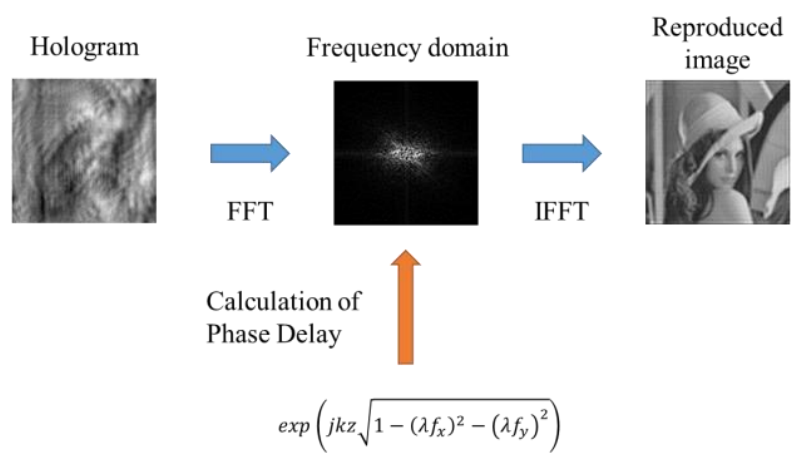

Fig. 2.2 Reconstruction of hologram.

First, the hologram is Fourier transformed, the phase delay amount is calculated in the frequency domain

A reconstructed image can be obtained by performing inverse Fourier transform on this

An error arises depending on the sampling points of the original image and the hologram, the interval, and the distance used for reconstruction.

\section{Iterative dummy area method}

\subsection{Dummy area method}

A dummy area method is one of methods for reducing the 
error of the reconstructed image. An area with an initial value of amplitude and phase of zero added to the periphery of the original image.

Assuming that the input image is $\boldsymbol{g}_{\mathbf{0}}(\boldsymbol{x}, \boldsymbol{y})$

In area of original image

$$
g_{0}(x, y)=\left|f_{0}(x, y)\right| \exp \left\{j \phi_{0}(x, y)\right\}
$$

In area of dummy area

$$
g_{0}(x, y)=0
$$

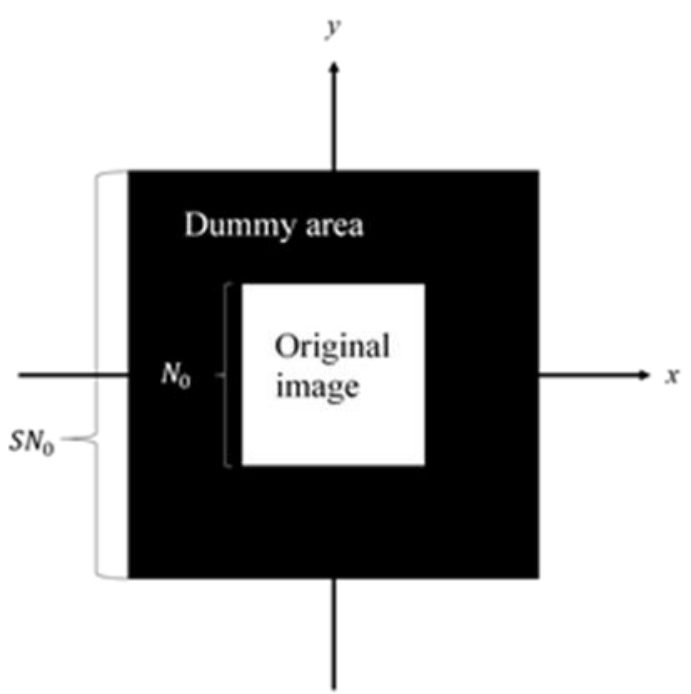

Fig. 3.1 Dummy area.

I will explain this $\mathrm{F}$ image as an original image

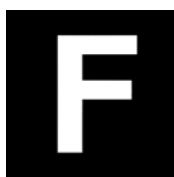

Fig. 3.2 Original image "F".

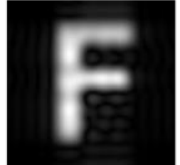

(a)No dummy area

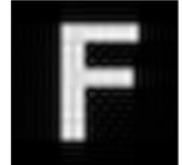

(b)Dummy area twice the original image

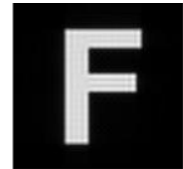

(c)Dummy area four times the original image
Fig. 3.3 Reconstructed image.

If a dummy area is used, the reconstructed image error can be reduced.

By this reason that adding dummy area is the order to be able to utilize the flexibility of the amplitude and phase.

\subsection{Problem of conventional dummy area method}

The dummy area method is equivalent to increasing the size of the original image. This also increases the size of the hologram. Data of the hologram is output to a highresolution printer, a spatial light modulator, or the like. The size of the hologram depends on the output device. Therefore, it is not desirable to increase the size of the hologram

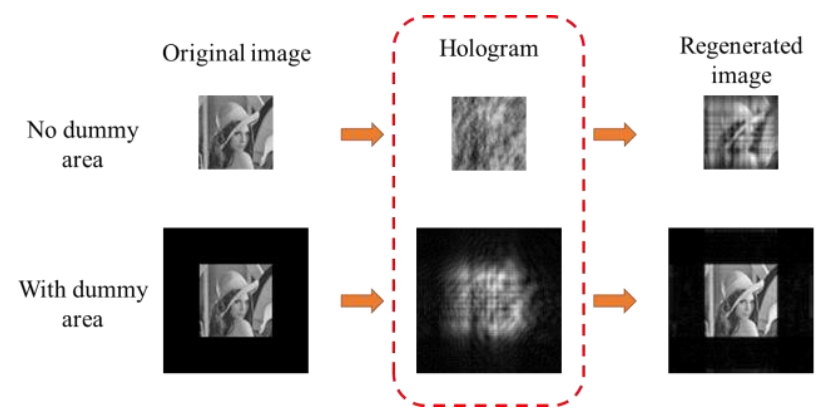

Fig. 3.4 conventional dummy area method.

\subsection{Proposed dummy area method}

In the proposed method, we stop using the whole area of the enlarged hologram for reconstruction. Cut the dummy area part of the hologram and use the hologram of the size of the original image before adding the dummy area for reconstruction.

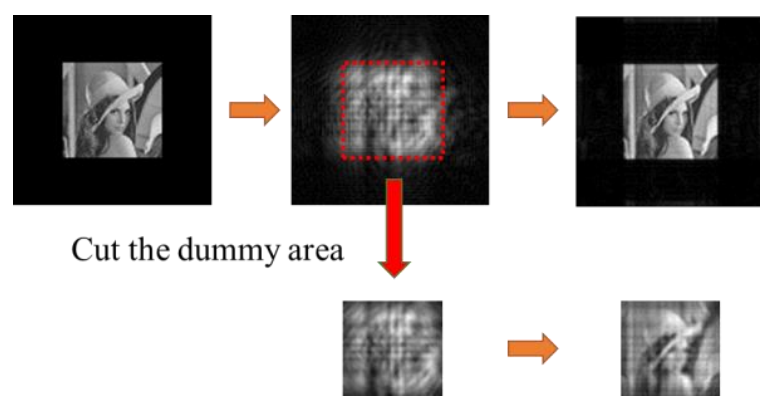

Fig. 3.5 Proposed dummy area method.

However, this method increases the error of the reconstructed image. This is because the dummy area in the hologram also contains information on the original image.

I will explain this image as an original image

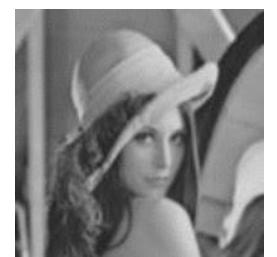

Fig. 3.6 Original image "Lenna" 


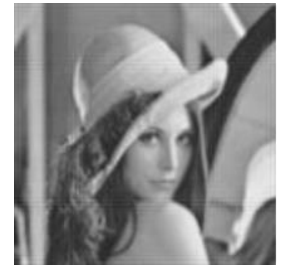

(a)Conventional dummy area method

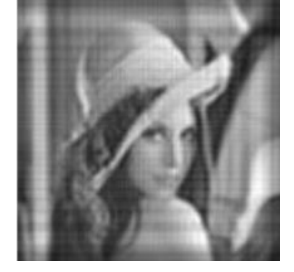

(b)Proposed dummy area method
Fig. 3.7 Reconstructed image.

If the dummy area is cut and reconstructed, the reconstructed image error increases and the error of the striped pattern is conspicuous in the reconstructed image.

\subsection{Iterative dummy area method}

In order to improve this result, I propose an iterative dummy area method.

At first, Cut the dummy area of the hologram and reproduce the whole area.

Next, Replace the original image area of the reconstructed image with the original image. Let this be the next input image.

By repeating this process, the distribution of the hologram is optimized

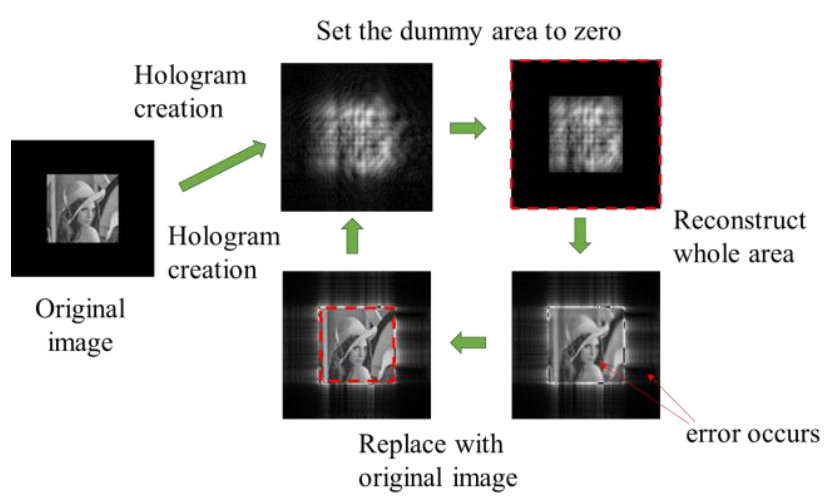

Fig. 3.8 Iterative dummy area method.

In the hologram created by this method, only the area of the original image can be used for reconstruction. It is possible to reduce the error without increasing the size of the hologram

\section{Simulation result}

Verify that the reconstructed image using the Iterative dummy area method can reduce errors generated by the proposed dummy area method.
To simulate with the same image.

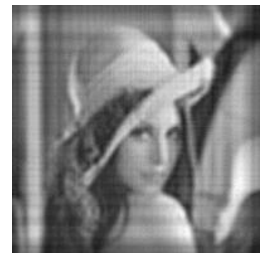

(a) Proposed

dummy area method

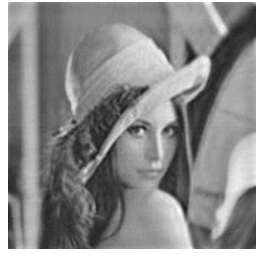

(b)Using iterative dummy area method

(1000 times)
Fig. 4.1 Reconstructed image.

It was found that it is possible to reduce the error by using the iterative dummy area method.

Next, we examine the effect of the number of iterations

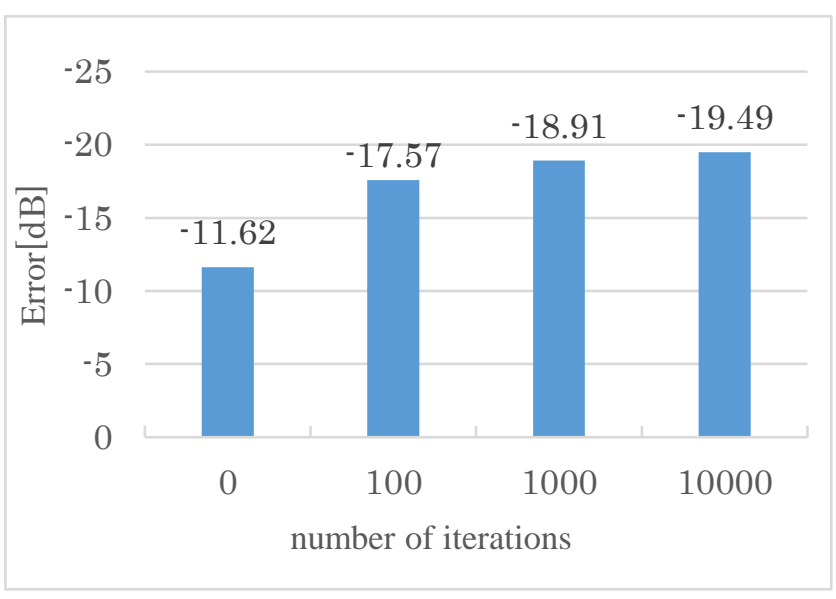

Fig. 4.2 Effect of the number of iterations.

In this graph, the larger the value, the more the error is reduced. It was found that the error can be further reduced by increasing the number of iterations.

\section{Conclusions}

We could reduce the error caused by proposed dummy area method by using iterative dummy area method. This made it possible to reduce the error without changing the size of the hologram.

\section{References}

(1) P. Harirahan, Principles of Holography, pp 1-13, pp 8894, (2004), Optronix Co

(2) Joseph W. Goodman, Fourier Optics (Third Edition), pp 287 - 288, pp 340 - 352, (2012), Mori Kita Publishing Co., Ltd. 